\section{Psychiatrie in der DDR II. Weitere Beiträge zur Geschichte}

Ekkehardt Kumbier (Hrsg.). Psychiatrie in der DDR II. Weitere Beiträge zur Geschichte. Schriftenreihe zur Medizin-Geschichte. Berlin: Be.bra wissenschaft, 2020, 432 Seiten, 32,00 Euro, ISBN 9783954102631

Als in der DDR ausgebildeter Nervenarzt und seit 1987 in der „Kinderneuropsychiatrie “ tätiger, wissenschaftlich und historisch orientierter Arzt und damit Teil des beschriebenen Systems hat mich die Fortsetzung der sehr fundierten und differenzierten Auseinandersetzung mit der Geschichte der Psychiatrie nach 1945 und dann speziell in der DDR in ihren Bann gezogen. Das ist zunächst dem Eingangskapitel, welches sich mit dem immer noch lesenswerten psychiatriekritischen Buch „Flucht in die Wolken“, das zu den illustrierenden Standardwerken in meinen studentischen Vorlesungen gehörte, und den fachlichen und politischen Reaktionen darauf beschäftigt, zu verdanken. Dass die insgesamt 21 Kapitel in dem 432 Seiten umfassenden Buch durch kinder- und jugendpsychiatrische Themen eingerahmt werden, zeugt davon, in welche Breite und Tiefe der Diskurs geht. Psychiatrie als ein mehr als andere medizinische Fächer gesellschaftlichen Erwartungen und Vorgaben ausgesetztes Fach bedarf der kritischen Rückschau, um reflektiert so unabhängig wie möglich seiner medizinischen und sozialen Verantwortung für psychisch kranke Menschen gerecht werden zu können. Diese Rückschau, zumindest was Entwicklungen in der DDR über 4 Jahrzehnte betrifft, ist mit diesem zweiten Band möglich.

Der Herausgeber hat den zweiten Band in 4 Abschnitte zum gesellschaftlich-politischen Kontext, zur Fächerdifferenzierung und Spezialisierung, zu diagnostischen und therapeutischen Ansätzen und zu Entwicklungen in einzelnen psychiatrischen Kliniken gegliedert. Bei den wissenschaftlichen Beiträgen handelt es sich um ein Konvolut, welches sowohl auf einzelne Persönlichkeiten wie Hans Szewczyk und Dietfried Müller-Hegemann, als auch auf Kliniken und Anstalten wie die Gerichtspsychiatrie der Charité, die Universitätsnervenklinik Jena, die Bezirksnervenklinik Ueckermünde, die Heil- und Pflegeanstalt Schwerin Sachsen- berg, auf die Entwicklung der Klinischen Psychologie, der Gerichtspsychiatrie und der Kinder- und Jugendpsychiatrie und deren Organisation in Fachgesellschaften, auf präventive und therapeutische Verfahren wie Telefonseelsorge, Aversionstherapie, Dialyse, Psychopharmakatherapie im Kontext industrieller Auftragsforschung, auf Ideologismen, auf die Wiederspiegelung der Psychiatrie im Filmmedium und auf den Missbrauch der Psychiatrie in der Sowjetunion fokussiert.

Der Band setzt mit einem sehr hohen Anspruch, dem er größtenteils gerecht wird, die im ersten Band begonnene, fundierte und differenzierte Auseinandersetzung mit der Psychiatriegeschichte nach 1945, speziell in der DDR fort. Neuere historische Forschungsarbeiten werden die oder den Herausgeber nötigen, in absehbarer Zeit diese jetzt schon verdienstvolle Reihe, um einen dritten Band zu erweitern.

Frank Häßler, Rostock

\section{Praxisbuch EEG. Grundlagen, Befundung, Beurteilung und differenzialdiagnostische Abgrenzung}

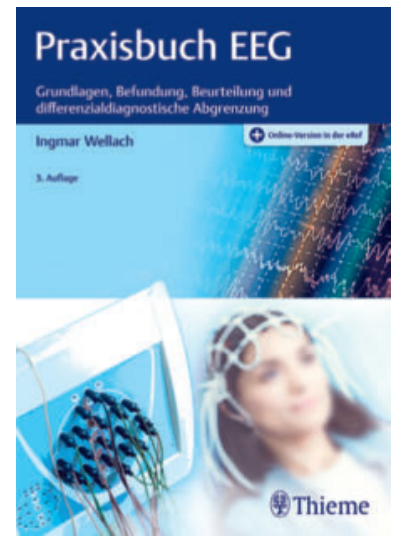

Ingmar Wellach (Hrsg.). Praxisbuch EEG. Grundlagen, Befundung, Beurteilung und differenzialdiagnostische Abgrenzung. Stuttgart: Thieme 2020, 608 Seiten, 238 Abbildungen, 3. Auflage, 99, 99 Euro, ISBN 9783132422070

Dieses Praxisbuch EEG möchte seinen Leser in die Grundlagen des EEG einführen und die möglichen Besonderheiten des EEG erläutern. Es ist ein Gemeinschaftswerk, wobei man sich wünschen würde, dass angegeben wird, welcher Autor welchen Abschnitt bearbeitet hat. Zu Beginn des Buches werden sehr ausführlich die elektrophysikalischen, anatomischen und physiologischen Grundlagen des EEG erläutert. Dieser Abschnitt ist sehr detailliert, er stellt eine exzellente Basis zum Verständnis des EEG dar, wäre aber für ein Praxisbuch weitgehend verzichtbar. In einem weiteren Abschnitt werden die technischen Grundlagen des EEG dargestellt. Hier erfährt der Leser ebenfalls sehr detailliert, welche physikalischen Prozesse zur eigentlichen EEG-Kurve führen. Es werden dann die normalen und abnormen EEG-Befunde erläutert. Dies geschieht in theoretischer Weise zumeist ohne entsprechende Abbildungen. Hier fragt sich der Leser, warum dieser Abschnitt eingefügt worden ist, wenn im darauffolgenden Teil des Buches die pathologischen Muster dargestellt werden. Im speziellen Teil werden die Anfallsmuster, die Muster bei Bewusstseinsstörungen und bei intensivmedizinischen $\mathrm{Er}$ krankungen sowie Medikamenteneffekte dargestellt. Es stellt sich die Frage, wie mit dem Routine-EEG, das in der normalen neurologischen Praxis abgeleitet wird, umgegangen werden soll. Ungewöhnlicherweise am Ende des Buches befindet sich ein historisches Kapitel über die Entstehung des EEG. Ein umfangreiches Literaturverzeichnis und ein brauchbares Sachverzeichnis runden das Buch ab.

Der Vorteil dieses Buches liegt sicherlich in der Fülle der Informationen von den feinsten elektrophysiologischen Grundlagen bis hin zu hochpathologischen EEG-Befunden. Dadurch ist die Handhabbarkeit etwas eingeschränkt, der Rückschluss von pathologischen Grafoelementen auf deren Nomenklatur und ätiologischer Grundlage ist nicht immer schnell nachvollziehbar. Ein weiterer Vorteil dieses Buches ist, dass es die modernen Klassifikationen und die moderne Terminologie für Epilepsie und für pathologische EEG-Elemente aufgreift. Es wird dabei aber auch deutlich, dass es leider immer noch keine weltweit verbindliche nachvollziehbare Terminologie und Strukturierung der EEG-Befundung gibt. Dennoch oder gerade deswegen handelt es sich um ein nützliches Buch, was auch darin zum Ausdruck gebracht wird, dass es jetzt in der dritten Auflage erscheint.

Stefan Evers, Coppenbrügge 